\title{
Enhancing accuracy and precision of transparent synthetic soil modelling
}

\section{Samuel A. Stanier}

Research Associate, Centre for Offshore Foundation Systems, University of Western Australia, Australia (Formerly PhD Research Student, University of Sheffield, UK)

\section{Jonathan A. Black}

Lecturer, Department of Civil and Structural Engineering, University of

Sheffield, UK

\section{Charles C. Hird}

Formerly Reader, Department of Civil and Structural Engineering, University of Sheffield, UK

Over recent years non-intrusive modelling techniques have been developed to investigate soil-structure interaction problems of increasingly complex geometry. This paper concerns the development of a small-scale, $1 \mathrm{~g}$, modelling technique using a transparent analogue for soil with particle image velocimetry for internal displacement measurement. Larger model geometry achieved in this research using fine-grained transparent synthetic soils has led to an increased need for rigorous photogrammetric correction techniques. A correction framework, based upon a modified version of the pinhole camera model, is presented that corrects for lens and camera movement induced errors as well as scaling from image space to object space. An additional statistical approach is also developed to enhance the system precision, by minimising the impact of increased non-coplanarity between the photogrammetry control plane and the target plane. The enhanced data correction and statistical precision is demonstrated using a case study examining the failure mechanism around a double helical screw pile installed in transparent synthetic soil representative of a soft clay.

\section{Notation}

$c_{\mathrm{V}}$

$D$

$e$

$\boldsymbol{g}$

$k$

$k_{1}$

$k_{2}$

$\mathrm{MB} / \mathrm{s}$

$m_{\mathrm{v}}$

$p_{1}$

$p_{2}$

$\mathbf{R}$

$s$ coefficient of consolidation

diameter

void ratio

gravitational acceleration

hydraulic conductivity

radial distortion parameter

radial distortion parameter

megabytes per second

coefficient of compressibility

tangential distortion parameter

tangential distortion parameter

radial distance

rotation matrix

helical plate spacing

undrained shear strength

translation vector

horizontal image space coordinate

horizontal image space coordinate corrected for internal camera errors

vertical image space coordinate corrected for internal and external camera errors

horizontal principal point

vertical image space coordinate

penetration velocity

vertical coordinate corrected for internal camera errors

$\begin{array}{ll}v_{\text {ie }} & \text { vertical image space coordinate corrected } \\ v_{0} & \text { for internal and external camera errors } \\ X & \text { vertical principal point } \\ Y & \text { horizontal object space coordinate } \\ z_{\text {ie }} & \text { vertical object space coordinate } \\ \alpha & \text { image space depth coordinate corrected for } \\ & \text { internal and external camera errors } \\ \delta_{\text {u:r }} & \text { aspect ratio } \\ \delta_{\text {u:t }} & \text { horizontal radial distortion error } \\ \delta_{\text {v:r }} & \text { horizontal tangential distortion error } \\ \delta_{\text {v:t }} & \text { vertical radial distortion error } \\ \kappa & \text { vertical tangential distortion error } \\ \lambda & \text { Eulerian rotation angle } \\ \varphi & \text { linear scaling ratio } \\ \omega & \text { Eulerian rotation angle } \\ & \text { Eulerian rotation angle }\end{array}$

\section{Introduction}

Transparent synthetic soils have been developed over the past 25 years to enable non-intrusive modelling of complex threedimensional geotechnical problems. Significant advantages of this technique compared to conventional plane strain physical modelling methodology are that it eliminates boundary effects encountered when testing against a rigid viewing window, reduces the need for geometrical model simplification and 
International Journal of Physical Modelling in Geotechnics Volume 12 Issue 4
Enhancing accuracy and precision of transparent synthetic soil modelling

Stanier, Black and Hird enables realistic processes to be modelled such as rotational installation.

A wide range of aggregate materials have been used as an analogue to represent coarse- and fine-grained soils which form a transparent continuum when blended with a pore fluid of matched refractive index. Coarse-grained soils reported in the literature include crushed borosilicate glass (Allersma, 1982), crushed Schott BK-7 optical glass (Konagai et al., 1992), silica gels (Ahmed and Iskander, 2011; Iskander et al., 2002b; Liu and Iskander, 2010; Sadek et al., 2002) and crushed quartz (Siemens et al., 2010). Fine-grained transparent soils have also been developed and two commonly referenced aggregates are precipitated amorphous silica (Hird et al., 2008; Iskander et al., 1994, 2002a; Liu et al., 2003; Ni et al., 2010; Sadek et al., 2002; Toiya et al., 2007; Welker et al., 1999) and fumed amorphous silica (Lehane and Gill, 2002; McKelvey et al., 2004). It is noteworthy that greater model geometries have been achieved using fine-grained soil owing to greater optical transparency. An associated benefit of testing fine-grained soils is that the mechanical behaviour is not stress dependent, thus representative failure mechanics can be observed at $1 \mathrm{~g}$. The work reported in this paper focuses on a fine-grained transparent medium that is representative of clay soil.

Recent advances in fine-grained transparent soil technology have harnessed modern image based deformation measurement capabilities using particle image velocimetry (PIV), specifically adapted for geotechnical applications as GeoPIV (White et al., 2003) or digital image correlation (DIC) (Sadek et al., 2003). Internal displacement measurement is achieved by tracking the trajectory of seeding particles that are illuminated using a laser sheet on a plane of interest within the soil volume, akin to PIV in the field of fluid mechanics. Applications in geotechnics using this methodology have included observations of installation disturbance around piles (Hird et al., 2008; Ni et al., 2010) and analysis of shallow footings (Iskander et al., 1994, 2002a; Liu et al., 2003; Sadek et al., 2002).

One of the limitations of these investigations is the accuracy and precision of the PIV measurements, as most of the investigations used a simple linear scale factor, $\lambda$, to account for conversion of measurements from image space to object space. Taylor et al. (1998) and White et al. (2003) demonstrated that application of rigorous photogrammetric correction, accounting for lens distortions or camera-soil movements, significantly improves the accuracy and precision of photographic displacement measurements.

Another limitation, noted by Ni et al. (2010), was that the scope of investigations possible using these techniques is often limited by the optical clarity of the transparent soil. However, Hird and Stanier (2010) appraised the consolidated transparency of the two most popular forms of fine-grained transparent soils (fumed and precipitated amorphous silica-based transparent soil), concluding that fumed silica soil allowed a doubling of the model size, while maintaining adequate PIV measurement precision. The increase in model size, thus increased noncoplanarity of the control plane used for photogrammetric correction and the target plane within the model on which PIV analysis is conducted, significantly reduces the precision of the photogrammetric correction system.

This paper describes experimental apparatus, modelling techniques and the development of a photogrammetric correction procedure that addresses this precision limitation. The photogrammetric correction procedure is based upon a modified version of the pinhole camera model (Heikkilä and Silvén, 1997), which accounts for lens-induced distortions and camera-soil movement-induced errors. A novel statistical precision enhancement is then proposed, which is capable of mitigating the negative impact of the increased control-target plane non-coplanarity on precision that is caused by increased model size. The success of the procedure is validated using a practical example where the failure mechanism of a double helical plate screw pile is observed. This application presented forms part of a larger body of work investigating helical screw piles owing to the considerable uncertainty among design engineers as to the actual failure mechanism that governs the capacity of multiple helical plate piles. The transparent soil allows observation of the failure mechanics, while facilitating realistic rotational or 'screwed' installation.

\section{Experimental procedure}

\subsection{Apparatus}

The experiments were performed in a black anodised (to minimise laser light reflections) aluminium test chamber (see Figure 1(a)) with transparent acrylic viewing windows in two adjacent faces: one to allow transmission of the laser light sheet through the soil model and the other to allow observation of soil movements on the plane of interest. Drainage was provided at the base of the chamber through three concentric semi-circular drainage rings overlain by a porous plastic filter.

Two detachable control point panels, each consisting of a row of eight red light emitting diodes (LED) at $20 \mathrm{~mm}$ spacings, were fixed to the front of the test chamber to provide a reference for photogrammetric correction. These were powered by a DC power supply voltage that was calibrated to provide optimum tracking of their positions using GeoPIV (Stanier, 2011).

Consolidation of the transparent soil from slurry was achieved using a Parker Hannifin PIE100-320 pneumatic piston that had a stroke length of $320 \mathrm{~mm}$ and was capable of delivering 
Enhancing accuracy and precision of transparent synthetic soil modelling Stanier, Black and Hird the required $100 \mathrm{kPa}$ vertical stress on a $200 \times 200 \mathrm{~mm}$ sample area. Drainage in the consolidation piston was provided in the same manner as in the base of the test chamber. The piston was sealed using a $4 \mathrm{~mm}$ cylindrical O-ring, mitred and bonded at $45^{\circ}$, to accommodate square fitment in the chamber corners.

A 1-watt argon-ion air-cooled laser (supplied by Laser Physics UK Ltd.) was used to produce a $0.95 \mathrm{~mm}$ diameter laser beam of 457-514 nm wavelength. The beam was passed through a top-hat beam shaper prism to produce a laser light sheet of approximately uniform intensity.

The model helical screw pile, seen in Figure 1(b), had a helical plate diameter of $20 \mathrm{~mm}$, shaft diameter of $5 \mathrm{~mm}$, helical plate pitch of $5 \mathrm{~mm}$, plate thickness of $1 \mathrm{~mm}$ and spacing of $30 \mathrm{~mm}$. The termination of the shaft was a $45^{\circ}$ cone to aid keying during installation. Installation was conducted at a screwed rate, whereby one revolution induced a vertical penetration of one flight pitch or $5 \mathrm{~mm}$. This was intended to minimise installation disturbance and was achieved using a twodimensional (2D) actuator (vertical displacement and rotation) capable of a maximum axial force of $\pm 1 \mathrm{kN}$, torque of $1 \mathrm{Nm}$ and an axial range of travel of $300 \mathrm{~mm}$ (see Figure 1(c)).
A Pentax K-10D digital single lens reflex (DSLR) camera was mounted upon a Manfrotto 055XPROB tripod and swivel ball head. Digital images were recorded on an 8 GB SanDisk Extreme III SD Memory card, with a maximum data transfer speed of $20 \mathrm{MB} / \mathrm{s}$, which facilitated continuous capture of 10 megapixel images at a rate of $3 \cdot 3$ frames per second.

\subsection{Transparent soil}

Hird and Stanier (2010) described in depth the transparent soil and transparency calibration used in this investigation. In brief the aggregate used was fumed silica powder (Wacker HDK-N20 supplied by Wacker Chemie AG) and the pore fluid was a matched refractive index blend of N-Paraffin C10-13 (supplied by Aztec Oils, Chesterfield, UK) and Baylube WOM 15 (supplied by Bayford Oils, Leeds, UK) in the ratio of 7723 by volume. The aggregates and matched refractive index pore fluid were mixed in the proportion of $6: 94$ by mass to create pourable slurry.

Following Ni et al. (2010), Timiron seeding powder consisting of platy mica particles $10-60 \mu \mathrm{m}$ in size coated in titanium oxide, were added in a proportion of $0.02 \%$ by mass with reference to the silica aggregate mass, to the transparent soil in the rear $52.5 \%$ of the chamber volume. The front $47.5 \%$ volume was left unseeded to preserve maximum transparency

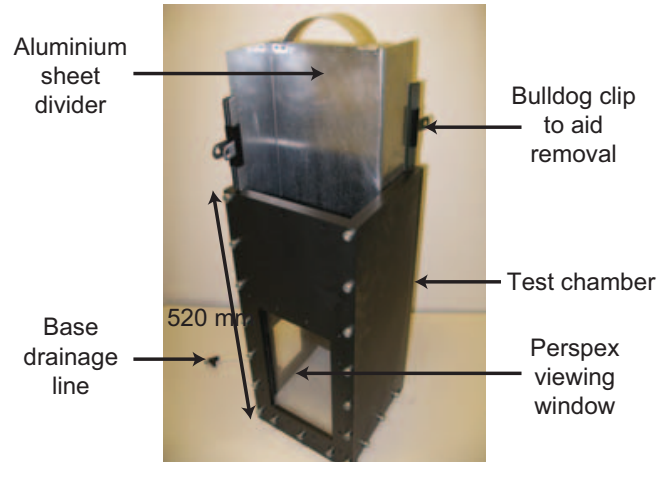

(a)

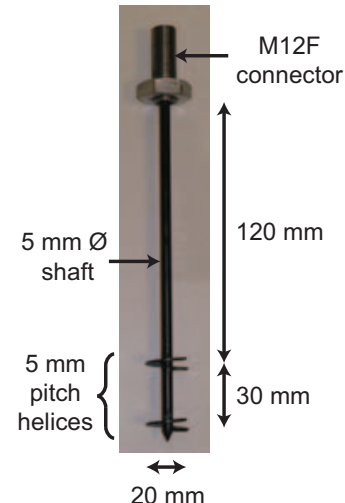

(b)

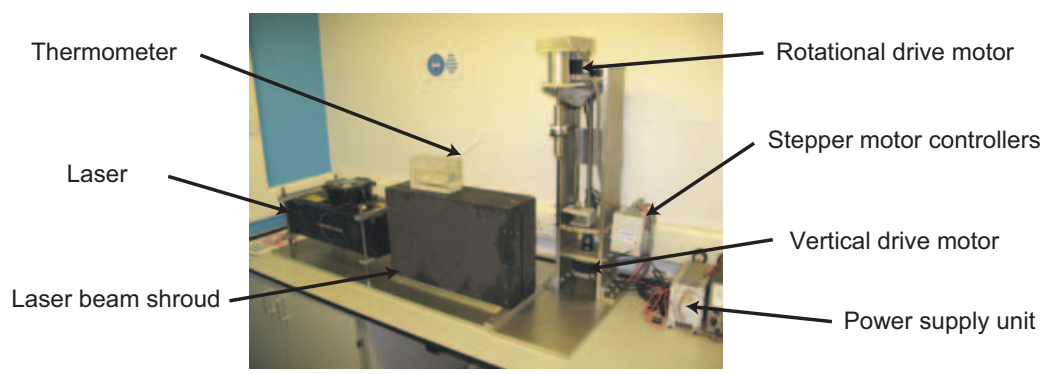

(c)

Figure 1. (a) Pile driving rig, (b) model double helix screw pile and

(c) test chamber 
International Journal of Physical Modelling in Geotechnics Volume 12 Issue 4
Enhancing accuracy and precision of transparent synthetic soil modelling

Stanier, Black and Hird in the foremost part of the model, through which the digital camera recorded soil movement on the plane of interest at the centre of the chamber. These particles, when illuminated by the laser light sheet, provide the distinct texture required for PIV displacement measurement (White et al., 2003) within the transparent soil.

\subsection{Model preparation}

All experimental procedures described here were conducted in an air-conditioned laboratory with the temperature maintained at $20^{\circ} \mathrm{C}$ throughout the testing process. After thorough mixing and de-airing of the transparent soil mix under vacuum, the slurry was poured into the test chamber using an aluminium sheet divider (see Figure 1(a)) to prevent mixing of the seeded and unseeded material. Once complete, the aluminium sheet divider was removed and the sample was consolidated in stages to an effective vertical stress of $100 \mathrm{kPa}$.

A draw-wire linear variable displacement transducer (LVDT) was used to monitor consolidation settlements, which allowed consolidation properties and other related parameters to be derived. Table 1 presents a summary of mean and standard deviations for the void ratio, $e$, coefficient of consolidation, $c_{\mathrm{v}}$, coefficient of compressibility, $m_{\mathrm{v}}$ and hydraulic conductivity, $k$, derived for the $50-100 \mathrm{kPa}$ effective stress increment, for a series of 12 samples with identical stress history. The small standard deviations indicate good sample preparation repeatability was achieved. The consolidation behaviour is similar to that of soft normally consolidated alluvial clays with compressibility characteristics comparable to very high organic content clays and peats (Gill, 1999). The undrained shear strength of the material, $s_{\mathrm{u}}$, following consolidation to a maximum effective vertical stress of $100 \mathrm{kPa}$ was measured using a $33 \mathrm{~mm}$ diameter hand vane, and was on average $15 \mathrm{kPa}$.

Upon completion of the consolidation process a small head of fluid was left on top of the sample to prevent air ingress during testing. Air-entrainment causes over-exposure in the digital images recorded by the camera and significantly degrades the precision of PIV tracking of soil movements.

\subsection{Testing}

The model pile was installed into the consolidated transparent soil model to a depth of 7D or $140 \mathrm{~mm}$, measured from the lowest mid helical plate depth. Following installation, a period of 24 hours was allowed prior to testing to facilitate equalisation of any installation induced excess pore pressures.

The test was conducted in a darkroom, with the laser light sheet illuminating the central plane of the model where the model pile was to be installed. The LED control point panels were energised and the digital camera was aligned using an alignment target such that the charge coupled device (CCD) within the camera was coplanar with the target plane. Images were captured by the digital camera at the maximum capture rate of $3 \cdot 3$ frames per second for the duration of the test, with camera settings of ISO $100, \mathrm{~F} 10 \cdot 0$ aperture and an exposure time of 1/10th of a second. Typically, during testing, a series of up to 70 images was recorded during mobilisation of the ultimate limit state (ULS).

The model pile was displaced at an undrained rate, $v$, by ensuring that the dimensionless velocity $\left(V=v D / c_{\mathrm{v}}\right)$ was greater than 30 (Finnie and Randolph, 1994), hence using the $c_{\mathrm{v}}$ values derived during sample preparation, a vertical drive speed of $0.2 \mathrm{~mm} / \mathrm{s}$ was selected.

\section{Data processing}

\subsection{PIV analysis}

All PIV analysis was conducted using GeoPIV (Take, 2003; White, 2002; White et al., 2003). The precision of PIV measurements computed by GeoPIV is highly dependent upon the discretisation of the problem. Large patches lead to close correlations but may lead to strain smearing in areas of strain localisation, whereas small patches leads to improved representation of localised strains at the expense of potentially poorer correlations (Take, 2003). It was demonstrated by Hird and Stanier (2010) that the standard errors yielded for fumed silica soil PIV tracking for patch sizes greater than 40 pixels were comparable to the upper bound estimator of White et al. (2003), which was fitted to calibration data derived from a real non-integer pixel displacement. This indicates that the texture provided by the Timiron is adequate to facilitate reasonably precise PIV tracking.

A patch size of 50 pixels was selected for the analysis presented in this work, giving a standard error of 0.012 pixels. The potential improvement in measurement precision for patch sizes greater than 50 pixels greatly reduces. For example using

\begin{tabular}{lcccc}
\hline Parameter & $e$ & $c_{\mathrm{v}}: \mathrm{m}^{2} /$ year & $m_{\mathrm{v}}: \mathrm{m}^{2} / \mathrm{kN}$ & $k: \mathrm{m} / \mathrm{s}$ \\
\hline Mean & 16.0 & 4.02 & $3.96 \times 10^{-3}$ & $4 \cdot 05 \times 10^{-9}$ \\
Standard deviation & 0.07 & 0.56 & $1.32 \times 10^{-4}$ & $5 \cdot 36 \times 10^{-10}$
\end{tabular}

Table 1. Soil parameters obtained during consolidation of test specimens 
Enhancing accuracy and precision of transparent synthetic soil modelling Stanier, Black and Hird the upper bound estimator of White et al. (2003), a 100-pixel patch potentially allows a $50 \%$ improvement in precision at the expense of a four-fold reduction in measurement density. Similarly using a 25-pixel patch potentially increases the measurement density by a factor of four at the expense of a $50 \%$ reduction in measurement precision. Clear representation of failure mechanisms in the form of displacement contours and shear strain fields requires both reasonable measurement density and high precision, thus a 50-pixel patch size was chosen as a compromise between maximising measurement density and maximising precision.

\subsection{Photogrammetric error correction}

Photogrammetric error consists of three key components: internal errors (camera lens induced: radial, tangential and pixel non-squareness distortions), external errors (cameratarget movements) and scale errors (conversion from image to object space units). In typical PIV investigations in plane strain chambers these can all be corrected in one step (White et al., 2003), so long as a series of image space and concurrent object space coordinates are available. However, owing to the need for the tests to be performed in a darkroom, LED control points have been used in lieu of traditional fixed optical targets, such as a black circle on a white background as used by White et al. (2003). This presented a significant obstacle to achieving satisfactory photogrammetric correction as calculating the centroid of LEDs in a digital image was deemed insufficiently accurate owing to flaring of the light. To counteract this, an alternative method was developed.

Taking the image space positions $u$ and $v$ in pixels of the PIV measurements of each patch at sequential time intervals, the errors due to radial ( $\delta_{\mathrm{u}: \mathrm{r}}$ and $\left.\delta_{\mathrm{v}: \mathrm{r}}\right)$ and tangential $\left(\delta_{\mathrm{u}: \mathrm{t}}\right.$ and $\left.\delta_{\mathrm{v}: \mathrm{t}}\right)$ lens distortion are expressed mathematically using Equations 1 and 2 where $k_{1}, k_{2}, p_{1}$ and $p_{2}$ are distortion coefficients. The radial distance, $r$, from the principal point, $u_{0}$ and $v_{0}$, is derived using Equation 3 (Slama, 1980).

1. $\left[\begin{array}{c}\delta_{\mathrm{u}: \mathrm{r}} \\ \delta_{\mathrm{v}: \mathrm{r}}\end{array}\right]=\left[\begin{array}{l}u\left(k_{1} r^{2}+k_{2} r^{4}\right) \\ v\left(k_{1} r^{2}+k_{2} r^{4}\right)\end{array}\right]$

2. $\quad\left[\begin{array}{c}\delta_{\mathrm{u}: \mathrm{t}} \\ \delta_{\mathrm{v}: \mathrm{t}}\end{array}\right]=\left[\begin{array}{c}2 p_{1} u v+p_{2}\left(r^{2}+2 u r^{2}\right) \\ p_{1}\left(r^{2}+2 v r^{2}\right)+2 p_{2} u v\end{array}\right]$

3. $r=\sqrt{\left(u-u_{0}\right)^{2}+\left(v-v_{0}\right)^{2}}$

CCD pixel non-squareness, which is typically linear and assumed to be constant over the area of the $\mathrm{CCD}$, is described mathematically by the aspect ratio, $\alpha$, which is the pixel height divided by the pixel width. Hence, after derivation of the calibration parameters $\left(u_{0}, v_{0}, k_{1}, k_{2}, p_{1}, p_{2}, \alpha\right)$, image space coordinates of measurement points can be corrected to generate coordinates adjusted for internal camera lens induced errors $\left(u_{\mathrm{i}}, v_{\mathrm{i}}\right)$ using Equation 4.

4. $\quad\left[\begin{array}{c}u_{\mathrm{i}} \\ v_{\mathrm{i}}\end{array}\right]=\left[\begin{array}{l}{\left[\frac{u-u_{0}}{\alpha}\right]-\left(\delta_{\mathrm{u}: \mathrm{r}}+\delta_{\mathrm{u}: \mathrm{t}}\right)} \\ \left(v-v_{0}\right)-\left(\delta_{\mathrm{u}: \mathrm{r}}+\delta_{\mathrm{u}: \mathrm{t}}\right)\end{array}\right]$

The investigation reported here was conducted at $1 \boldsymbol{g}$ hence it was deemed satisfactory to perform one single camera calibration at the beginning of the testing schedule based on the assumption that the camera settings (focal length, shutter duration, aperture, ISO, zoom length and position) remain constant between successive images and tests. Take (2003) noted that camera calibration parameters can vary significantly during centrifuge testing due to flexibility of the lens in the high $\boldsymbol{g}$ environment, thus the photogrammetric correction technique described here is best suited to $1 g$ applications.

The correction framework used to remove camera-target movement errors (Equation 5) is a modified form of the pinhole camera model, which was proposed by Heikkilä and Silvén, 1997) and utilised by White et al. (2003). This framework consists of a transformation matrix incorporating a normalised camera matrix, a rotation matrix, $R$ (Equation $6)$, described in terms of Eulerian rotation angles $(\varphi, \omega, \kappa)$ (Equation 7) and a translation vector, $t$ with components of displacement for the three principal axes $\left(t_{x}, t_{y}, t_{z}\right)$ (Equation $8)$. The terms equal to unity in the left- and right-hand side vectors are used to express the coordinate positions of the points of interest in homogeneous terms. The rotation matrix and translation vector describe the projection path of a point on the plane of interest, through the camera focal point, onto the CCD of the camera. The use of a normalised camera matrix (where the focal length is assumed to be equal to unity) means that the photogrammetric control points (in this case the two columns of LEDs) are not used to derive a scale factor, $\lambda$, to convert the measurements from image space to object space. Thus Equation 5 is used to convert image space coordinates corrected for lens induced distortions $\left(u_{\mathrm{i}}, v_{\mathrm{i}}\right)$ to image space coordinates corrected for lens induced distortions and differential camera-target movement errors $\left(u_{\mathrm{ie}}, v_{\mathrm{ie}}\right)$.

5. $\quad\left[\begin{array}{l}u_{\mathrm{i}} \\ v_{\mathrm{i}} \\ 1\end{array}\right]=\left[\begin{array}{llll}1 & 0 & 0 & 0 \\ 0 & 1 & 0 & 0 \\ 0 & 0 & 1 & 0\end{array}\right]\left[\begin{array}{ll}R & t \\ 0 & 1\end{array}\right]\left[\begin{array}{l}u_{\mathrm{ie}} \\ v_{\mathrm{ie}} \\ z_{\mathrm{ie}} \\ 1\end{array}\right]$ 
International Journal of Physical Modelling in Geotechnics Volume 12 Issue 4
Enhancing accuracy and precision of transparent synthetic soil modelling Stanier, Black and Hird
6. $R=\left[\begin{array}{lll}r_{11} & r_{12} & r_{13} \\ r_{21} & r_{22} & r_{23} \\ r_{31} & r_{32} & r_{33}\end{array}\right]$

$r_{11}=\cos \varphi \cos \kappa$

$r_{12}=-\sin \kappa \cos \omega+\cos \kappa \sin \varphi \sin \omega$

$r_{21}=\sin \kappa \cos \varphi$

7.

$r_{22}=\cos \kappa \cos \omega+\sin \kappa \sin \varphi \sin \omega$

$r_{23}=-\cos \kappa \sin \omega+\sin \kappa \sin \varphi \cos \omega$

$r_{31}=-\sin \varphi$

$r_{32}=\cos \varphi \sin \omega$

$r_{33}=\cos \varphi \cos \omega$

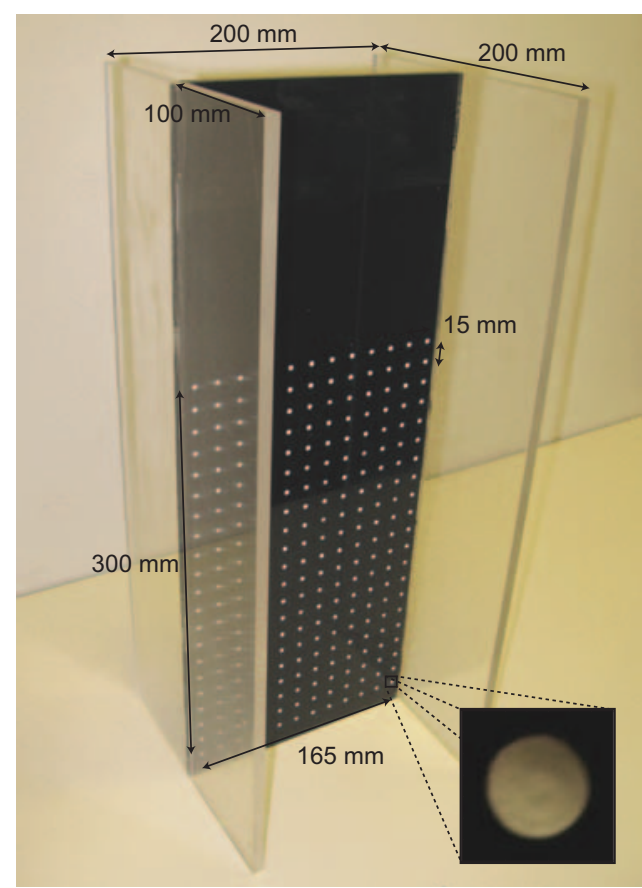

Figure 2. Photogrammetric correction calibration panel and subset zoom of drilled control point, back filled with white correction fluid

backfilled with white correction fluid. The calibration panel was placed coincident with the target plane inside the test chamber, which was flooded with the mineral oil pore-fluid to mimic the test conditions (given that the transparent soil and oil based pore fluid have matched refractive indices). The LED control point panels were attached to the front wall of the test chamber and the digital camera was setup allowing a subset of 98 visible control points to be used for the system validation.

The measurement data are converted from image space, corrected for internal and external camera induced errors, $u_{\mathrm{ie}}$ and $v_{\text {ie, }}$, to object space coordinates $X$ and $Y$, using a linear scale factor, $\lambda$. Typically this scale factor would be calculated using the control points (White et al., 2003), however, in this investigation the position of the tip of the model pile at commencement of the installation process and upon completion, is used to derive the scale factor. Owing to the pile being on the target plane, this scale factor accounts for both image space to object space scaling and refraction scaling caused by the transparent acrylic viewing window and the $100 \mathrm{~mm}$ depth of transparent soil.

\section{System validation}

\subsection{Procedure}

A calibration panel, with $2523 \mathrm{~mm}$ diameter blind holes drilled at $15 \mathrm{~mm}$ centres to an accuracy of $10 \mu \mathrm{m}$ was manufactured (see Figure 2) and used to validate the proposed photogrammetric correction process. For maximum contrast the calibration plane was painted matt black and the blind holes were were recorded at the maximum capture rate of the digital camera. The initial image space centroids of each of the visible control points were derived using a weighted average centroiding process documented in detail in Stanier (2011). Following this the control points were tracked through the series of images using GeoPIV, which was more precise than the centroiding process initially used to derive the centroid locations.

Relative movement of the camera and model was tracked using GeoPIV by tracking the position of each LED control point using a 100-pixel patch (there was no penalty for using large patch sizes owing to the black background of the control panels) thus replicating the test arrangement. The LED movements were then used in the proposed photogrammetric correction procedure to correct the apparent movement of the control points on the target plane in the centre of the chamber. The
Seventy images of the calibration panel within the test chamber 
International Journal of Physical Modelling in Geotechnics Volume 12 Issue 4
Enhancing accuracy and precision of transparent synthetic soil modelling Stanier, Black and Hird known movement of the target plane control points was zero; hence any discrepancy provided a measure of the system error.

The camera calibration parameters $\left(u_{0}, v_{0}, k_{1}, k_{2}, p_{1}, p_{2}, \alpha\right)$ were derived using a camera calibration toolbox for Matlab ${ }^{\mathrm{TM}}$ (Heikkilä, 2000). This was achieved using six images of the same calibration panel (except that it was not surrounded by the test chamber and all 252 control points were used) taken at various angles at a similar distance from the camera to the test scenario. The same aforementioned centroiding procedure was used to generate image space locations for each of the control points.

\subsection{Summary of precision}

Table 2 provides a summary of the precision of each of the calculation processes incorporated into the proposed photogrammetric correction procedure. As can be seen the least precise process is calculation of the camera calibration parameters, which are used to correct image space data for lens induced distortions. This likely limits the accuracy of the system, as is demonstrated in the following section.

\section{Results}

Control point movements on the target plane, corrected using the described photogrammetric correction procedure, were used to assess the impact validity of the procedure. Visual inspection of vectorial error plots over the field of view showed no systematic pattern to the residual errors (see Stanier, 2011). As systematic errors are indicative of inadequate correction of lens distortions (White et al., 2003) the corrections applied were deemed to be performing adequately.

Figure 3(a) presents the horizontal and vertical residual errors for each control point, with standard deviations of $38.0 \mu \mathrm{m}$ and $65.0 \mu \mathrm{m}$ respectively. These errors were larger than those quoted by White (2002) of $16 \cdot 7 \mu \mathrm{m}$ system accuracy but an improvement on simple linear scaling, for which an accuracy of $107.6 \mu \mathrm{m}$ was quoted. Figure $3(\mathrm{~b})$ is a plot of the temporal evolution of the standard deviation of the positional errors, which appear to be stable, confirming that the correction of lens induced distortions, is precise. Of greater importance are the mean residual errors presented in Figure 3(c). As the control points are stationary with relation to the LED control points and test chamber during calibration, this graphically represents the camera-soil movement errors that are not removed by the external correction process (Equation 5). The maximum error in either horizontal or vertical component is approximately $25 \mu \mathrm{m}$. Hence, if the minimum displacement contour plotted is $25 \mu \mathrm{m}$, then it can only be stated with confidence that it is representative of a displacement magnitude of $25 \pm 25 \mu \mathrm{m}$, rendering contours of displacement of this magnitude meaningless.

Table 2 indicated that the transparent soil tracked more accurately than the control points on the calibration target, thus it was necessary to investigate the residual camera-soil movement errors for transparent soil. Two samples were used for this purpose and GeoPIV was used to track the movement of 98 randomly positioned 50-pixel patches throughout a series of 70 images where zero soil movement occurred. The mean residual errors in Figure 4(a) are very similar to those observed in the validation using the calibration panel; however, in Figure 4(b) they are much larger potentially owing to local vibrations in an adjacent laboratory. It was important to ascertain why the mean error was not zero as was expected, given that camera-soil movement errors were corrected using Equation 5.

In typical plane strain and axisymmetric imaging applications the control point plane is coincident with the exposed plane of

Component of measurement system

Precision

\begin{tabular}{|c|c|c|}
\hline component ol measurement system & Image space: pixels & Object space: $\mu m^{a}$ \\
\hline Transparent soil tracking using GeoPIV & $0 \cdot 012$ & 0.77 \\
\hline $\begin{array}{l}\text { Calibration panel control point location by way of } \\
\text { centroiding }\end{array}$ & 0.05 & $3 \cdot 25$ \\
\hline $\begin{array}{l}\text { Calibration panel control point tracking using } \\
\text { GeoPIV }\end{array}$ & 0.02 & $1 \cdot 29$ \\
\hline LED tracking using GeoPIV & 0.065 & $4 \cdot 19$ \\
\hline Camera calibration procedure ${ }^{b}$ & $0 \cdot 5$ & $32 \cdot 3$ \\
\hline
\end{tabular}

${ }^{a}$ Calculated using an approximate linear scale factor of $115 \cdot 5$ (mm/pixel).

${ }^{\mathrm{b}}$ Mean of horizontal and vertical precisions presented.

Table 2. Summary of precision of individual processes incorporated into the proposed photogrammetric correction procedure 


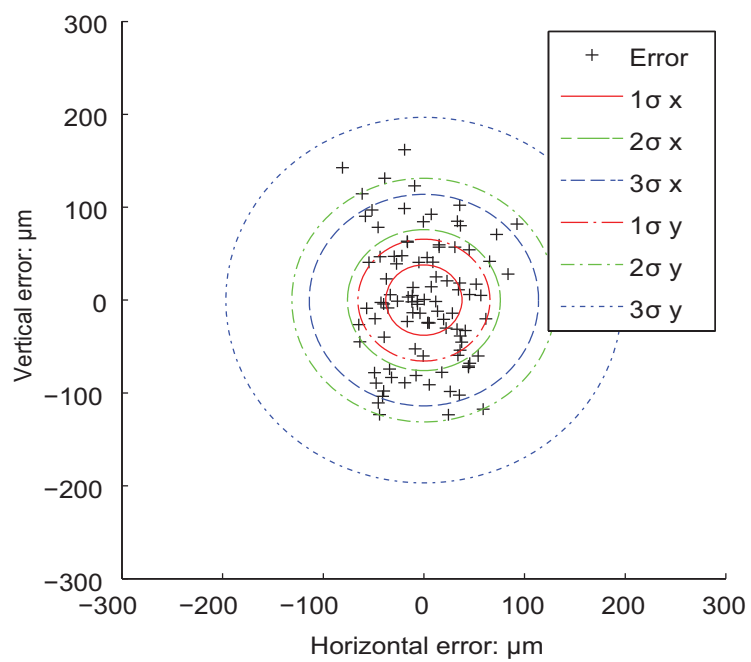

(a)

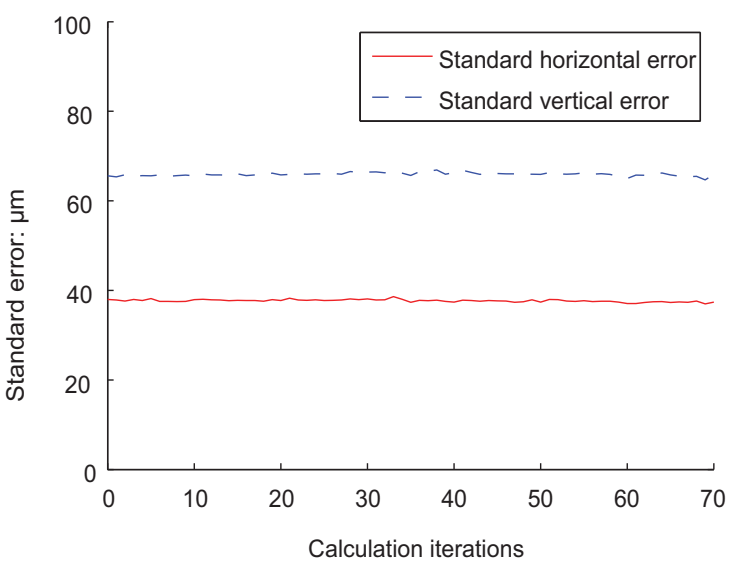

(b)

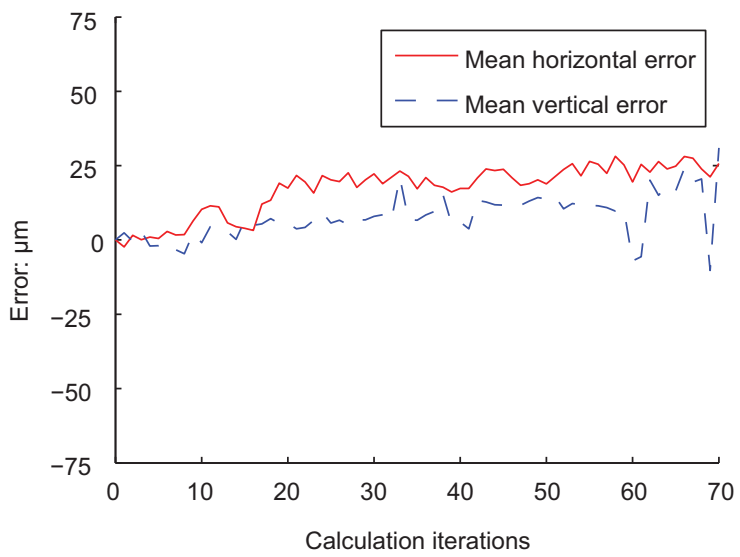

(c)

Figure 3. Plots of residual errors distribution and standard deviations after photogrammetric correction for (a) internal, camera lens induced distortions, (b) standard errors and (c) mean errors over 70 calculation iterations

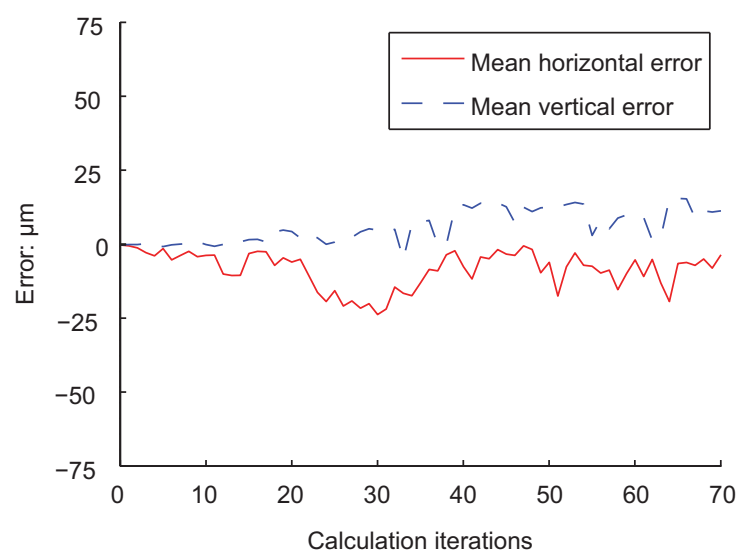

(a)

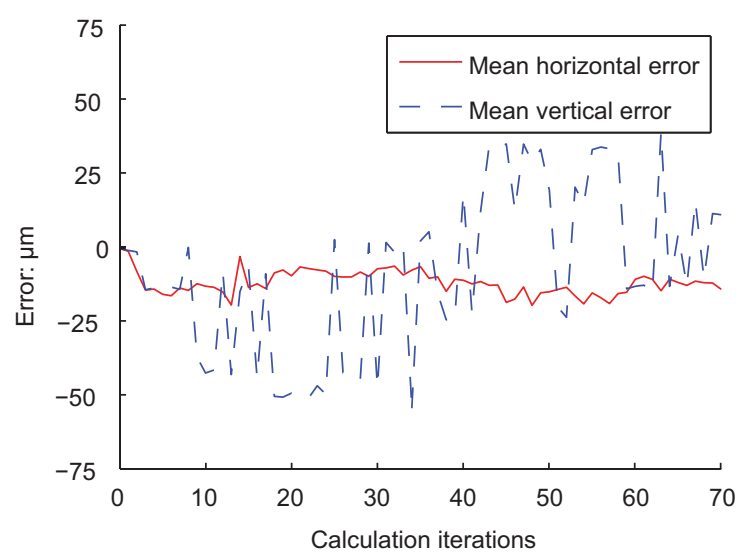

(b)

Figure 4. Plots of mean residual errors for (a) first zero soil movement analysis and (b) second zero soil movement analysis

soil. In this investigation the distance between the control point plane (LEDs) and the target plane (centre of the model) is a distance of $140 \mathrm{~mm}$. It is this increased non-coplanarity between the control point plane and target plane that is the source of the errors seen in Figure 3(c) and Figure 4(a) and 4(b). It was not possible to place the control points on the measurement plane for two reasons: first, control points cannot be spatially spread across the field of view within the model (as commonly deployed on the visible soil surface in plane strain models) and second, they would prevent the laser light sheet uniformly penetrating the sample producing a loss of particle illumination. Due to the lack of reliable image space and object space coordinates for the LED control points, it was impossible to create a closed-form correction to account for the non-coplanarity, even though the perpendicular distance between the control point and target planes was known. Hence, an alternative, indirect method of accounting for this non-coplanarity was developed. 


\subsection{Statistical precision enhancement}

In the three validation analyses presented here zero displacement is evident on the plane of interest. Hence, the modal magnitudes of the mean residual errors could be used to correct the data, as the modal magnitude should be identical to the camera-target movement error. Fortunately, this technique can also be used for a physical model test assuming that

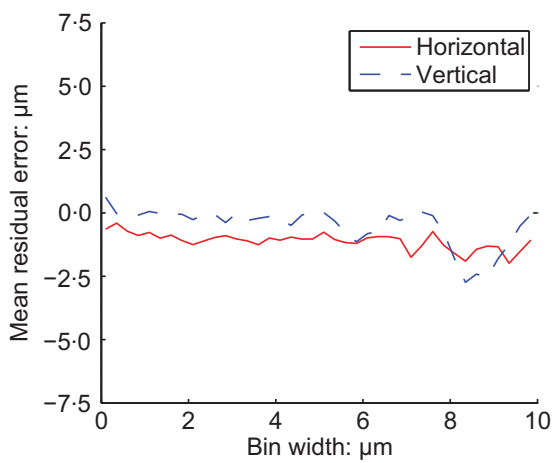

(a)

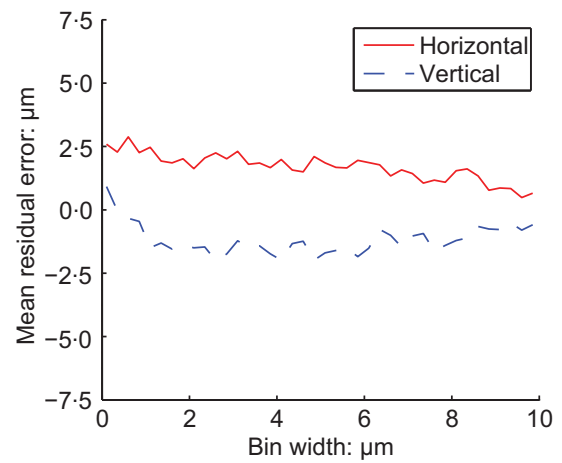

(c)

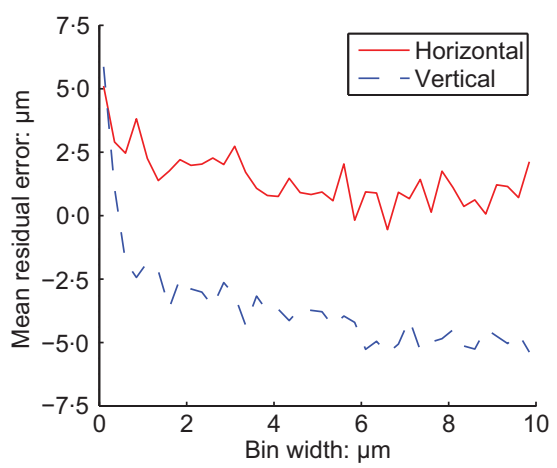

(e)

Figure 5. Mean and maximum absolute residual error with increasing histogram bin width from modal movement correction for the calibration panel (a-b) and zero soil movement analysis one (c-d) and two (e-f) respectively (a) the boundary effects are minimised by ensuring there is sufficient free boundary distance

(b) displacement of the geotechnical structure is used to induce displacements within the soil continuum

(c) the surface area of the geotechnical structure is significantly smaller than the surface area of the boundary of the physical model

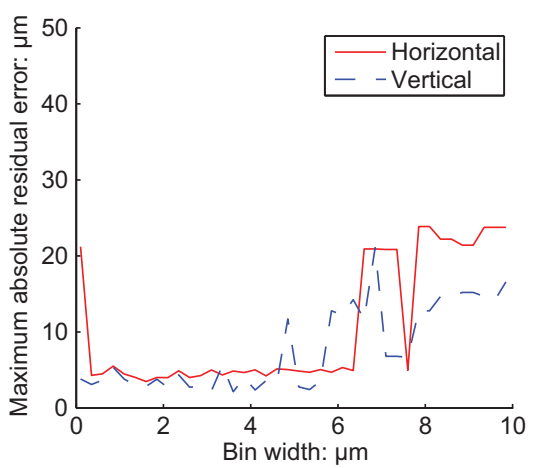

(b)

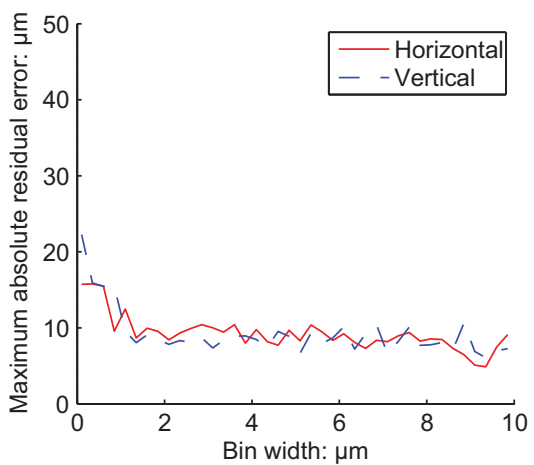

(d)

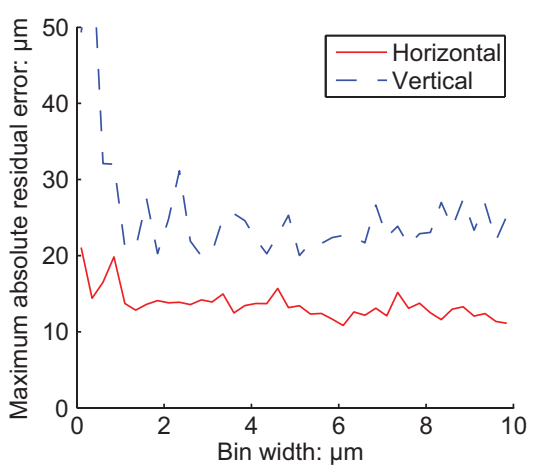

(f) 
(d) PIV analysis patches are uniformly spaced throughout the model and encompass areas of the model where zero soil displacement is anticipated.

If these constraints are met, the gradient of displacement within the soil continuum is maximal at the interface with the geotechnical structure that is applying displacement to the soil and minimal where there is zero soil movement. In these circumstances, it may be assumed that modal magnitudes of horizontal and vertical displacement will be equal to the

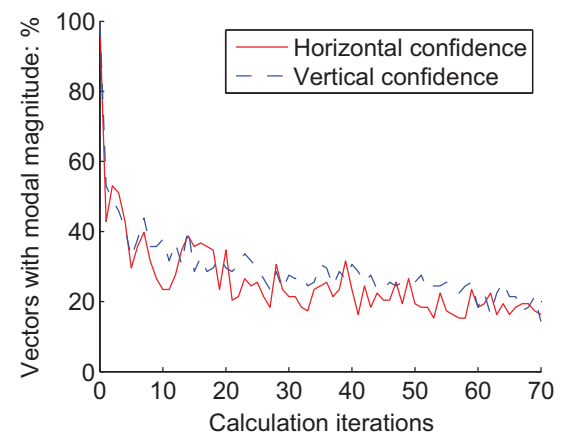

(a)

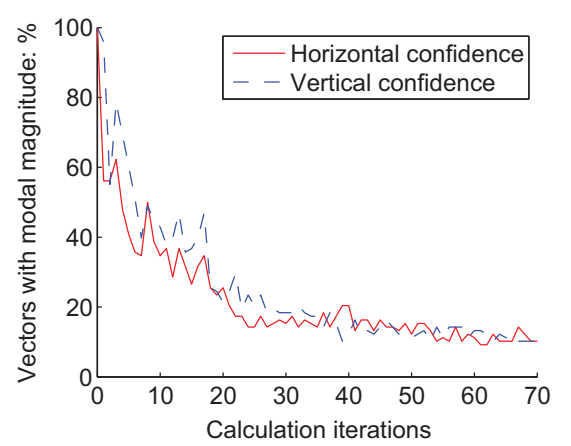

(c)

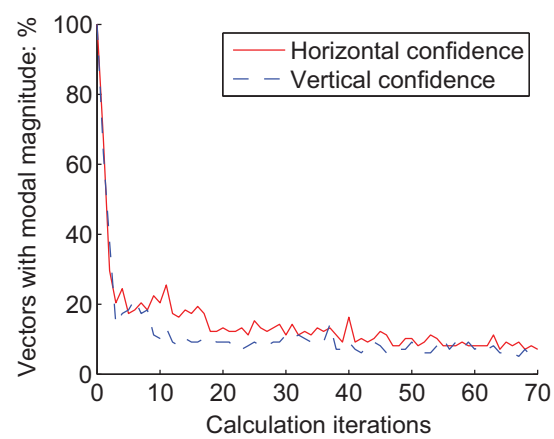

(e)

Figure 6. Percentage of vectors with modal magnitude and residual mean error after application of modal correction for the calibration panel $(a-b)$ and zero soil movement analysis one $(c-d)$ and two (e-f) respectively residual camera-target movement error, since the modal magnitude is most likely to fall at the minimum gradient of displacement. These modal magnitudes can be calculated for each image in the PIV analysis process and removed from all measurement points to generate data with reduced cameratarget movement errors.

A simple histogram is used to calculate the modal magnitude for each displacement component. The histogram bin width is the only subjective choice and is selected such that the modal

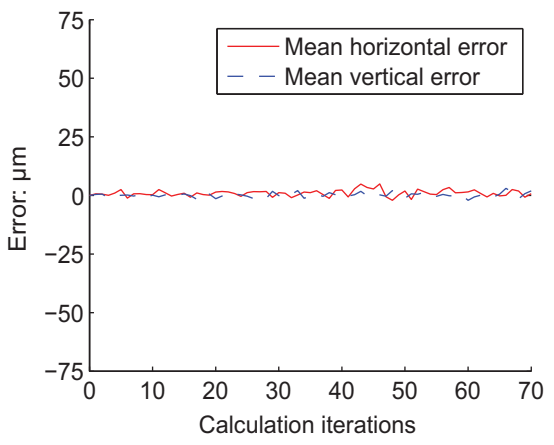

(b)

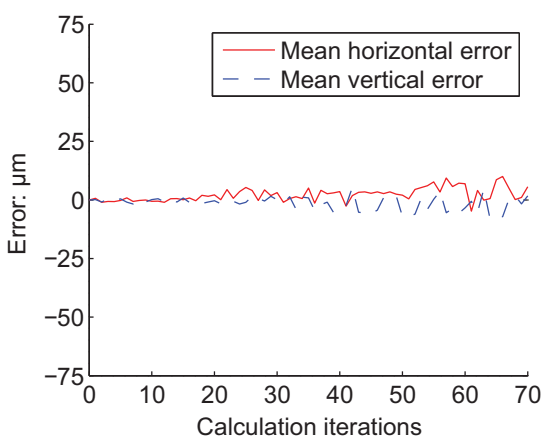

(d)

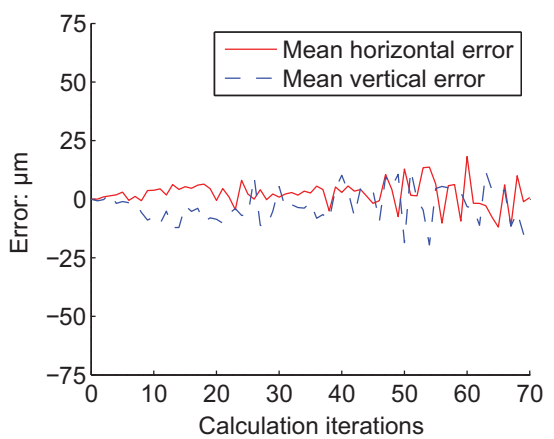

(f) 
Enhancing accuracy and precision of transparent synthetic soil modelling Stanier, Black and Hird bin has a frequency that is significantly greater than those of the other bins. Figure 5 presents the mean and maximum absolute residual error after correction for all control points or soil patches from the calibration target, first and second zero soil movement validations, with increasing histogram bin width for all 70 images used in each case. The data presented show that the optimum bin width for this investigation is $2 \mu \mathrm{m}$, as it is the smallest bin width for which both the mean and maximum absolute residual errors remain small in all three cases. This maximises the resolution of the enhancement, which is equivalent (using the approximate linear scale factor) to 0.03 pixels. Therefore, the statistical enhancement approach proposed here is more accurate than simply tracking the movement of the rigid horizontal and vertical boundaries of the test chamber manually, which would have a maximum resolution of 1 pixel, or $65 \mu \mathrm{m}$. It is important to note that the choice of bin width is unique to each experimental set-up and requires similar calibration procedures to be performed, so the value utilised here is specific to this investigation.

\section{Impact on system precision}

Figure 6(a), (c) and (e) show the percentage of vectors equal to the modal magnitude and provides an indication of confidence

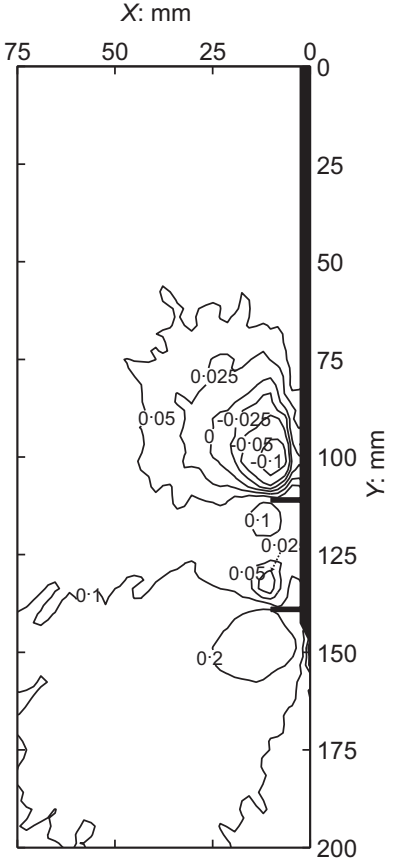

(a)

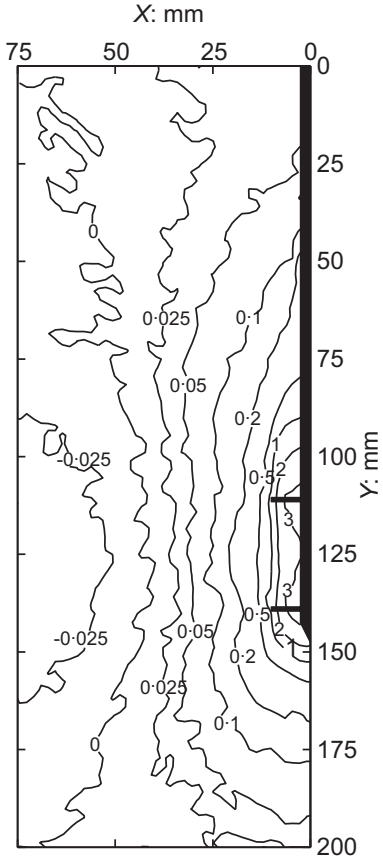

(b) in the correction. In all three cases the percentage of measurement points equal to the modal magnitudes reduces rapidly to a steady $10-20 \%$ due to random walk errors in GeoPIV (White, 2002). In an actual test, where the soil is experiencing differential displacements induced by a geotechnical structure, this percentage is typically lower. The effect that this statistical precision enhancement has on the photogrammetric correction system precision is quite dramatic, as demonstrated by the data in Figure 6(b), (d) and (f). The average residual error presented in these three validations is reduced to $5-20 \mu \mathrm{m}$ and thus the enhanced precision of the system is closer to that reported by White (2002).

The application of this statistical precision enhancement can also be observed when used on actual test data. Figure 7(a) and (b) are cumulative horizontal and vertical contour plots prior to application of the modal correction, from a double helix pile test with helical plate spacing of $30 \mathrm{~mm}$, at a tensile displacement of $3 \mathrm{~mm}$. Positive displacement magnitudes indicate upward and rightward displacement. The horizontal contours presented in Figure 7(a) are clearly unreasonable, since the zero contour above the upper helical plate would indicate that almost all of the soil is moving rightwards to some degree. Given the relatively deep embedment ratio of $4 \mathrm{D}$, a more symmetrical

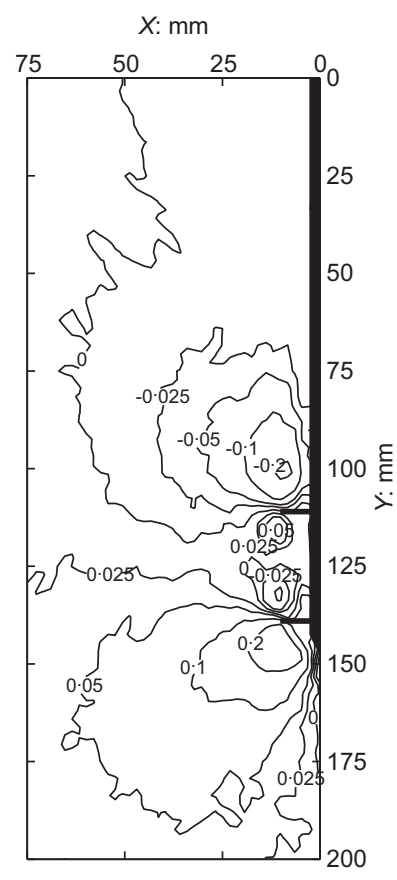

(c)

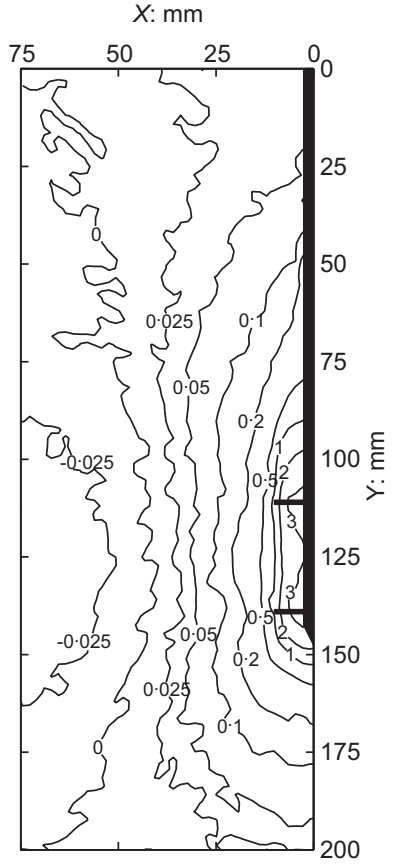

(d)

Figure 7. Displacement contours at $3 \mathrm{~mm}$ tensile displacement for a double helix pile with $30 \mathrm{~mm}$ spacing: (a) uncorrected horizontal and (b) vertical, (c) displacement and corrected horizontal and (d) vertical displacement, with displacements in $\mathrm{mm}$ 
horizontal displacement field was expected with the zero horizontal displacement contour lying between the helical plates. The vertical contours in Figure 7(b) are more reasonable.

Using the statistical precision enhancement method proposed, the horizontal error was calculated to be $67 \mu \mathrm{m}$, with $4 \cdot 1 \%$ of the vectors exhibiting the modal magnitude. Similarly, the vertical correction was calculated to be $0 \mu \mathrm{m}$, with $2 \cdot 41 \%$ of the vectors being equal to the modal magnitude. This indicates that the test system was potentially subjected to some horizontal vibration during testing. Figure 7(c) and (d) present the corrected horizontal and vertical contours. These plots indicate that the corrected horizontal contour plot is now more reasonable as the zero contour emanates from between the two helical plates and propagates towards the horizontal boundary. Because the vertical correction to be applied was $0 \mu \mathrm{m}$, the vertical contour remains unchanged, thus the method is suitable for precautionary use in instances where no correction is necessarily required.

\section{Example test output}

Figure 8 presents the vectorial displacement (a), horizontal contour plot (b), vertical contour plot (c) and incremental engineering shear strain (d), showing the failure mechanism for a $1 \mathrm{~mm}$ increment of displacement at the ULS (defined using the load-deflection response measured using a load-cell), for a double helix pile with helical plate spacing, $s$, of $30 \mathrm{~mm}$.

In Figure 8 (a) the vectorial displacements are shown scaled by a factor of 5 , indicating that in many instances vectorial displacement plots in isolation are insufficient when assessing failure mechanisms of complex geotechnical structures.

The horizontal and vertical contour plots in Figures 8(b) and (c) respectively, which are dependent upon the modal magnitude movement mitigation technique proposed here, allow better appraisal of the failure mechanism. The failure mechanism appears to be deep, since the mechanism is symmetrical about the mid-height of the active length of the pile (the zone between the uppermost and lowermost helical plates) and the horizontal component of displacement is far smaller than the vertical component.

In the engineering shear strain plot in Figure 8(d) a distinct cylindrical shear band is shown to have formed at the outer radii of the helical plates. It should be noted that the shear strain fields are unaffected by the modal movement precision

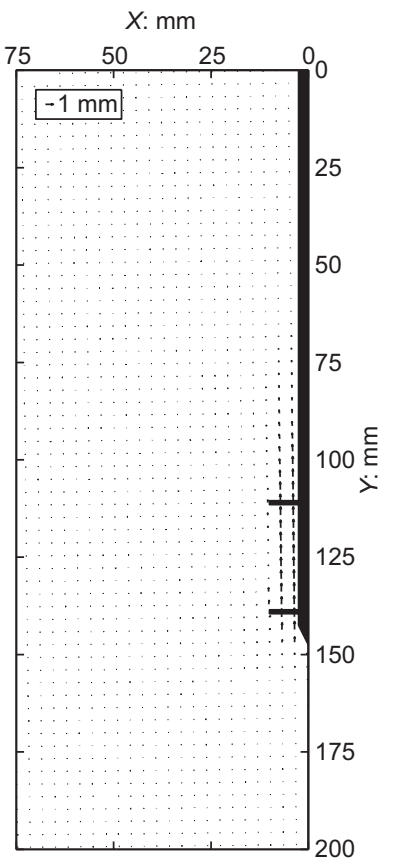

(a)

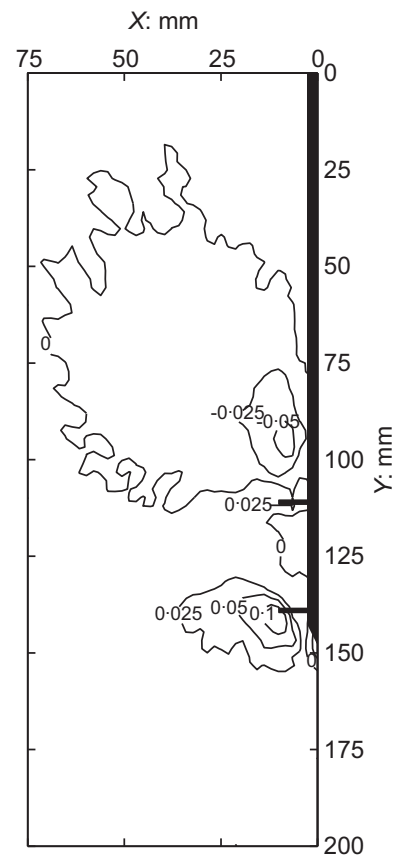

(b)

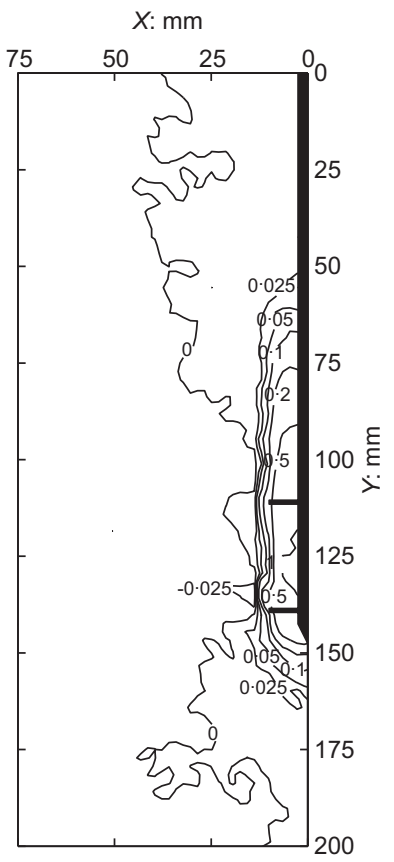

(c)

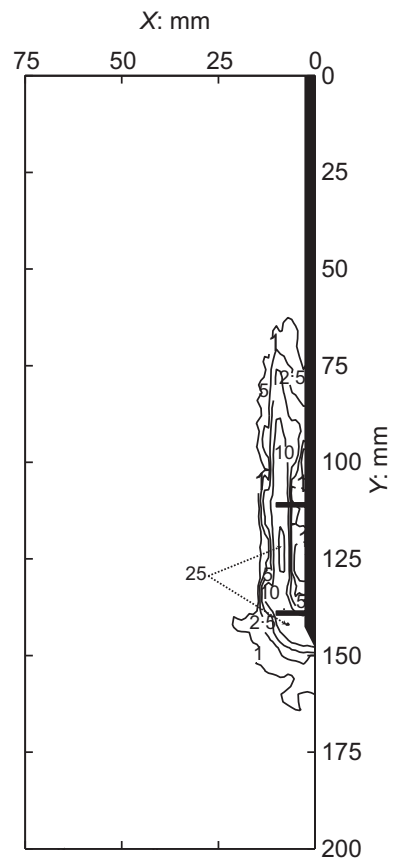

(d)

Figure 8. Double helix pile with $30 \mathrm{~mm}$ spacing incremental PIV results from $4 \mathrm{~mm}$ to $5 \mathrm{~mm}$ axial tensile displacement: (a) vector, (b) horizontal displacement, (c) vertical displacement and (d) shear strain contours with displacements in $\mathrm{mm}$ and shear strains expressed as a percentage 
International Journal of Physical Modelling in Geotechnics Volume 12 Issue 4
Enhancing accuracy and precision of transparent synthetic soil modelling Stanier, Black and Hird enhancement procedure since shear strains are derived from the first derivative of the displacements, hence systematic errors in the displacement components have no impact on the calculated strains. These observations validate the theory proposed by Rao et al. (1993), that a cylindrical failure mechanism is prevalent for helical screw piles with small helical plate spacing ratios ( $s / D$ of $1 \cdot 5$ or less).

\section{Conclusions}

The research presented in this paper aimed to develop a photogrammetric correction procedure, facilitating accurate and precise measurement of soil deformations non-intrusively within a body of transparent soil. Results emanating from this aim lead to the following conclusions.

1. A rigorous photogrammetric correction procedure, suitable for use with non-intrusive modelling techniques involving transparent soils at $1 \boldsymbol{g}$, has been presented. This procedure accounts for radial, tangential and pixel non-squareness lens-induced distortions as well as camera-target movements and scaling from image space to object space.

2. The precision of the photogrammetric correction procedure has then been enhanced using a statistical approach to mitigate systematic (translational) errors due to controltarget plane non-coplanarity. This procedure has been demonstrated significantly to improve system precision, such that the output is comparable in precision to that achieved by White (2002) in a plane strain chamber with much less significant control-target plane non-coplanarity.

3. An example analysis, of the failure mechanism of a double helical screw pile, has been used to demonstrate the validity and merits of the modelling procedure. Highresolution displacement fields were attained showing the displacement of soil around the helical pile at the ULS. These results together with the associated shear strain fields indicate that the failure mechanism for this closely spaced helical pile $(s / D=1 \cdot 5)$ features generation of a cylindrical shear failure surface at the outer radii of the helical plates.

\section{Acknowledgements}

The authors are grateful to the technical staff at the University of Sheffield for their able assistance in performing the experiments presented in this paper, and to the authors of GeoPIV for allowing use of their software in the investigations. Dr V. Sivakumar, Queen's University Belfast is also acknowledged for providing fumed silica for this study.

\section{REFERENCES}

Ahmed M and Iskander M (2011) Analysis of tunnelling-induced ground movements using transparent soil models. Journal of Geotechnical and Geoenvironmental Engineering, ASCE 137(5): 525-535.
Allersma H (1982) Photoelastic investigation of the stress distribution during penetration. Proceedings of 2 nd European Symposium on Penetration Testing, Amsterdam, the Netherlands, pp. 411-418.

Finnie IMS and Randolph MF (1994) Punch-through and liquefaction induced failure of shallow foundations on calcareous sediments. Proceedings of International Conference on Behaviour of Offshore Structures, Boston, USA, pp. 217-230.

Gill D (1999) Experimental and Theoretical Investigations of Pile and Penetrometer Installation in Clay. PhD thesis, Trinity College, Dublin, Ireland.

Heikkilä J (2000) Camera Calibration Toolbox for Matlab ${ }^{T M}$ Version 3.0. University of Oulu, Finland. See http://www. ee.oulu.fi/ jth/calibr (accessed 15/11/2012).

Heikkilä J and Silvén O (1997) A four-step camera calibration procedure with implicit image correction. Proceedings of the 1997 Conference on Computer Vision and Pattern Recognition, San Juan, Puerto Rico, pp. 1106-1112.

Hird C and Stanier SA (2010) Modelling helical screw piles in clay using a transparent soil. Proceedings of the 7 th International Conference on Physical Modelling in Geotechnics, Zurich, Switzerland, pp. 769-774.

Hird C, Ni Q and Guymer I (2008) Physical modelling of displacements around continuous augers in clay. Proceedings of 2nd British Geotechnical Association International Conference on Foundations, Dundee, UK, vol. 1, pp. 565-574.

Iskander M, Lai J, Oswald C and Mannheimer R (1994) Development of a transparent material to model the geotechnical properties of soils. Geotechnical Testing Journal 17(4): 425-433.

Iskander M, Liu J and Sadek S (2002a) Transparent amorphous silica to model clay. Journal of Geotechnical and Geoenvironmental Engineering 128(3): 262-273.

Iskander M, Sadek S and Liu J (2002b) Optical measurement of deformation using transparent silica gel to model sand. International Journal of Physical Modelling in Geotechnics 2(4): 13-126.

Konagai K, Tamura C, Rangelow P and Matsushima T (1992) Laser aided tomography: A tool for visualization of changes in the fabric of granular assemblage. Structural Engineering/Earthquake Engineering 9(3): 193-201.

Lehane B and Gill D (2004) Displacement fields induced by penetrometer installation in an artificial soil. International Journal of Physical Modelling in Geotechnics 4(1): 25-36.

Liu J and Iskander M (2010) Modelling capacity of transparent soil. Canadian Geotechnical Journal 47(4): 451-460.

Liu J, Iskander M and Sadek S (2003) Consolidation and permeability of transparent amorphous silica. Geotechnical Testing Journal 26(4): 1-12.

McKelvey D, Sivakumar V, Bell A and Graham J (2004) Modelling vibrated stone columns in soft clay. Proceedings of the 
Institution of Civil Engineers - Geotechnical Engineering 157(3): 137-149.

Ni Q, Hird C and Guymer I (2010) Physical modelling of pile penetration in clay using transparent soil and particle image velocimetry. Géotechnique 60(2): 121-132.

Rao S, Prasad Y and Veeresh C (1993) Behaviour of embedded model screw anchors in soft clays. Géotechnique 43(4): 605 614.

Sadek S, Iskander M and Liu J (2002) Geotechnical properties of transparent silica. Canadian Geotechnical Journal 39(1): 111-124.

Sadek S, Iskander M and Liu J (2003) Accuracy of digital image correlations for measuring deformations in transparent media. Journal of Computing in Civil Engineering 17(2): 88-96.

Siemens G, Peters S and Take WA (2010) Analysis of a drawdown test displaying the use of transparent soil in unsaturated flow applications. Proceedings of 5th International Conference on Unsaturated Soils, Barcelona, Spain, pp. 733 738.

Slama C (1980) Manual of Photogrammetry, 4th edn. American Society of Photogrammetry: Falls Church, VA, USA.
Stanier SA (2011) Modelling the Behaviour of Helical Screw Piles. PhD thesis, University of Sheffield, UK.

Take W (2003) The Influence of Seasonal Moisture Cycles on Clay Slopes. PhD thesis, University of Cambridge, UK.

Taylor R, Grant R, Robson S and Kuwano J (1998) An image analysis system for determining plane and $3 \mathrm{D}$ displacements in soil models. In Centrifuge '98: Proceedings of the International Conference, IS-Tokyo '98 (Kimura T, Kusakabe $\mathrm{O}$ and Takemura $\mathrm{J}$ (eds)). Taylor \& Francis, Abingdon, UK, vol. 1, pp. 73-78.

Toiya M, Hettinga J and Losert W (2007) 3D imaging of particle motion during penetrometer testing: From microscopic to macroscopic soil mechanics. Granular Matter 9(5): 323-329.

Welker A, Bowders J and Gilbert R (1999) Applied research using a transparent material with hydraulic properties similar to soil. Geotechnical Testing Journal 22(3): 266-270.

White D (2002) An Investigation into the Behaviour of Pressed-in Piles. PhD thesis, University of Cambridge, UK.

White D, Take W and Bolton M (2003) Soil deformation measurement using particle image velocimetry (PIV) and photogrammetry. Géotechnique 53(7): 619-631.

\section{WHAT DO YOU THINK?}

To discuss this paper, please email up to 500 words to the editor at journals@ice.org.uk. Your contribution will be forwarded to the author(s) for a reply and, if considered appropriate by the editorial panel, will be published as discussion in a future issue of the journal.

International Journal of Physical Modelling in Geotechnics relies entirely on contributions sent in by civil engineering professionals, academics and students. Papers should be 2000-5000 words long (briefing papers should be 10002000 words long), with adequate illustrations and references. You can submit your paper online via www.icevirtuallibrary.com/content/journals, where you will also find detailed author guidelines. 